\title{
Ischaemic lateral popliteal nerve palsy due to ergot intoxication
}

\author{
G. D. PERKIN \\ From the National Hospitals for Nervous Diseases, Maida Vale Hospital, London
}

SYNOPSIS A patient with ergot intoxication due to medication for migraine developed lateral popliteal nerve palsy. This is attributed to recurrent ischaemia caused by ergotamine.

Although the development of diffuse peripheral paraesthesiae and numbness is a recognized complication of ergot intoxication, an isolated lateral popliteal nerve palsy has not been described previously in this situation. The purpose of this paper is to record such a case in a patient with evidence of severe arterial spasm in the lower limbs, and to point to some analogies with those cases of isolated lateral popliteal palsies secondary to atherosclerotic disease.

\section{CASE REPORT}

The patient (MV 76725), a Greek woman aged 30 years, was first admitted in August 1973. She had a four year history of migraine, responding poorly to ergotamine tartrate, $2 \mathrm{mg}$ (Lingraine) and clonidine. For three months she had been using Cafergot suppositories (ergotamine tartrate $2 \mathrm{mg}$, caffeine $100 \mathrm{mg}$, belladonna alkaloids $0.25 \mathrm{mg}$, isobutylallyl barbituric acid $100 \mathrm{mg}$ ) up to four times in an attack and 12 times per week.

One week before admission, and 24 hours after using suppositories, she awoke with an intense burning pain, associated with numbness, over the dorsum of the right foot and had difficulty in moving the toes. The symptoms persisted.

On admission, the peripheries were warm, and all pulses were present. The blood pressure was 140/90 mmHg. Neurological examination showed abnormalities confined to the right leg, where there was impaired sensation to all modalities over the dorsum of the foot, and slight weakness of dorsiflexion of the foot and hallux. The tendon reflexes were present and symmetrical.

The following investigations were normal-full blood count, fasting blood sugar, serum urea, electrolytes, cholesterol, uric acid, calcium, phos- phorus, and alkaline phosphatase. Successive erythrocyte sedimentation rates (ESR) were 28, 32, 36 , and $30 \mathrm{~mm}$ in the first hour. The cerebrospinal fluid had no cells, and a protein content of 50 $\mathrm{mg} / \mathrm{dl}$. Serology was negative. Radiography of the chest and lumbar spine, and myelography were normal. On electromyography, performed at normal skin temperature, the distal motor latency to extensor digitorum brevis on stimulation of the right lateral popliteal nerve was $4.3 \mathrm{~ms}$ with motor nerve conduction velocity (knee-ankle) $48 \mathrm{~m} / \mathrm{s}$. The amplitude of the muscle action potential from stimulation at the knee was $100 \mu \mathrm{V}$ and from the ankle, $250 \mu \mathrm{V}$. The ascending nerve action potential, recording with needle electrodes at the knee and stimulating at the ankle, was absent. Distal motor latency to extensor digitorum brevis on stimulating the left lateral popliteal nerve was $3.5 \mathrm{~ms}$ with conduction velocity (knee-ankle) $50 \mathrm{~m} / \mathrm{s}$. The amplitude of the evoked muscle action potential from stimulation at the knee was $5 \mathrm{mV}$ and from the ankle $6 \mathrm{mV}$. The ascending nerve action potential had an amplitude of $12 \mu \mathrm{V}$, with a latency to peak of $7 \mathrm{~ms}$.

During this first admission the right foot was noted to be occasionally warmer than the left. Intradermal acetylcholine produced normal responses.

After discharge from hospital the pain lessened, but the paraesthesiae and numbness persisted and were exacerbated by crossing the right leg over the left.

On 24 September the patient used four more suppositories and woke the following day with severe pain in the right leg from the foot to the knee anteriorly, increased numbness, and periodic waves of burning discomfort radiating up the right leg. She was admitted two days later, having used three further suppositories that morning.

Examination showed normal upper limb pulses with a blood pressure of $160 / 100 \mathrm{mmHg}$. In the 
lower limbs, all pulses below the femorals were absent, apart from equivocal foot pulses on the left. The right leg was cold below the knee with cyanosis and pallor on elevation. The left leg was affected similarly, but less severely.

Neurological abnormalities were again confined to the right leg. A more extensive area of impaired sensation was present over the dorsum of the foot, extending anterolaterally towards the knee, and in addition to weakness of dorsiflexion of foot and hallux there was slight weakness of plantar flexion. The tendon reflexes were preserved.

The following investigations were normal-full blood count, blood urea, electrolytes, protein electrophoresis, and immunoglobulins. Serial ESRs were 32 and $11 \mathrm{~mm} / \mathrm{hr}$. Cryoglobulins, cold agglutinins, and antinuclear factor were not detected in the blood. Electromyography of the right tibialis anterior muscle (recorded when skin temperature had returned to normal) showed no spontaneous activity and no increase in insertional activity. The interference pattern was not assessed because pain prevented full muscular contraction. In the right lateral popliteal nerve the distal motor latency to extensor digitorum brevis was $5 \mathrm{~ms}$ with a conduction velocity (knee-ankle) of $23 \mathrm{~m} / \mathrm{s}$. The amplitude of the evoked muscle action potential from stimulation at the knee was $80 \mu \mathrm{V}$, and from the ankle 100 $\mu \mathrm{V}$. The ascending nerve action potential was again absent.

Ergot preparations were withdrawn and within 48 hours the skin temperature was normal and peripheral pulses had returned apart from the right popliteal pulse. Subsequently the pain remitted, but the sensory impairment remained, though to a lesser degree. After discharge from hospital, the patient noticed an aching discomfort with burning in the leg, precipitated by walking and relieved by rest. In December 1973 stimulation of the right lateral popliteal nerve disclosed a distal motor latency to extensor digitorum brevis of $5.7 \mathrm{~ms}$ with a conduction velocity (knee-ankle) of $34 \mathrm{~m} / \mathrm{s}$. The amplitude of the evoked response from stimulation at the knee was $260 \mu \mathrm{V}$ and from the ankle $310 \mu \mathrm{V}$. The nerve action potential remained absent.

\section{DISCUSSION}

The diagnosis of ergot intoxication was established by the rapid return of peripheral pulses after withdrawal of the drug. The elevated ESR suggested alternative diagnoses but this finding has been recorded before in ergotism (Horton and Peters, 1963; Glazer et al., 1966).

Previous reports of neurological complica- tions from ergot intoxication have described peripheral, symmetrical, sensory loss and paraesthesiae, often with spontaneous pain during recovery (Wilson, 1954; Horton and Peters, 1963; Glazer et al., 1966; Yao et al., 1970). In some respects, these cases resemble the ischaemic neuritis described in atherosclerotic peripheral vascular disease (Mufson, 1952; Hutchinson and Liversedge, 1956; Garven et al., 1962; Eames and Lange, 1967), the pathological features of which are predominantly large fibre demyelination and remyelination with varying degrees of axonal degeneration (Garven et al., 1962; Eames and Lange, 1967).

In this case, however, despite the eventual evidence of generalized arterial insufficiency of the lower limbs, the clinical features pointed to a relatively selective lateral popliteal nerve ischaemia, and a similar selectivity has been reported in other vascular disorders affecting the lower limbs.

Where the peripheral pulses have been normal -as, for example, in a case of temporal arteritis with lateral popliteal palsy described by Ross Russell (1959) - occlusion of nutrient arteries to the nerve has been held responsible. Though ligation of single nutrient arteries to the sciatic nerve in dogs failed to produce clinical manifestations or pathological change, ligation of multiple nutrient arteries or embolization of a single artery produced both (Roberts, 1948). In cases of polyarteritis nodosa, involved peripheral nerves tend to show occlusion of multiple nutrient arteries (Kernohan and Woltman, 1938; Richards, 1951) and Roberts (1948) has described a patient with subacute bacterial endocarditis and a lateral popliteal palsy who showed, at postmortem examination, obstruction of the nutrient arteries by embolus, with thrombosis of the vasa nervorum in the middle of the nerve.

With occlusive disease of the femoral or popliteal arteries due to arteriosclerotic thrombosis or embolism, Mufson (1952) and Ferguson and Liversedge (1954) have demonstrated an increased susceptibility of the lateral popliteal nerve to the resulting ischaemia, though the findings of depressed ankle jerks or weakness of plantar flexion in some of these cases have shown that this increased susceptibility is relative rather than absolute.

In this patient, the site of vascular obstruc- 
tion, clinically, was between the femoral and popliteal arteries. The superficial femoral artery appears to be the most commonly affected vessel in cases of ergotism which have been investigated angiographically (Glazer et al., 1966; Fagerberg et al., 1967; Yao et al., 1970).

Sensory symptoms and signs predominated in this case, in contrast with the findings in palsies secondary to thrombosis or embolism. Conceivably, the chronic repetitive ischaemia of the nerve reproduced the features described with generalized ischaemic neuritis, and certainly the marked slowing of conduction velocity which eventually developed would favour the presence of segmental demyelination.

Sunderland (1945) has investigated the blood supply of the nerves of the lower limb. Within the sciatic nerve there appears to be no difference in the blood supply of the medial and lateral popliteal divisions. At the level of the popliteal fossa, however, though the medial popliteal nerve receives several nutrient arteries directly from the popliteal artery, the lateral popliteal nerve receives at most one direct nutrient artery, relying on indirect supply from the musculocutaneous or superior lateral genicular branches of the popliteal artery.

It thus seems likely, as Ferguson and Liversedge (1954) have suggested, that this segment of the lateral popliteal nerve is more at risk from developing ischaemia with obstructive lesions of the femoral or popliteal arteries than either the sciatic nerve itself or its medial popliteal division.

I would like to thank Dr R. S. Kocen for permission to publish details of a case under his care.

\section{REFERENCES}

Eames, R. A., and Lange, L. S. (1967). Clinical and pathological study of ischaemic neuropathy. Journal of Neurology, Neurosurgery, and Psychiatry, 30, 215-226.

Fagerberg, S., Jorulf, H., and Sandberg, C. G. (1967). Ergotism, arteriospastic disease and recovery, studied angiographically. Acta Medica Scandinavica, 182, 769-772.

Ferguson, F. R., and Liversedge, L. A. (1954). Ischaemic lateral popliteal nerve palsy. British Medical Journal, 2, 333-335.

Garven, H. S. D., Gairns, F. W., and Smith, G. (1962). The nerve fibre populations of the nerves of the leg in chronic occlusive arterial disease in man. Scottish Medical Journal, 7, 250-265.

Glazer, G., Myers, K. A., and Davies, E. R. (1966). Ergot poisoning. Postgraduate Medical Journal, 42, 562-568.

Horton, B. T., and Peters, G. A. (1963). Clinical manifestations of excessive use of ergotamine preparations and management of withdrawal effect: report of 52 cases. Headache, 2, 214-227.

Hutchinson, E. C., and Liversedge, L. A. (1956). Neuropathy in peripheral vascular disease. Quarterly Journal of Medicine, 25, 267-274.

Kernohan, J. W., and Woltman, H. W. (1938). Periarteritis nodosa. Archives of Neurology and Psychiatry (Chic.), 39, 655-686.

Mufson, I. (1952). Diagnosis and treatment of neural complications of peripheral arterial obliterative disease. Angiology, 3, 392-396.

Richards, R. L. (1951). Ischaemic lesions of peripheral nerves: a review. Journal of Neurology, Neurosurgery, and Psychiatry, 14, 76-87.

Roberts, J. T. (1948). The effect of occlusive arterial diseases of the extremities on the blood supply of nerves. Experimental and clinical studies on the role of the vasa nervorum. American Heart Journal, 35, 369-392.

Russell, R. W. R. (1959). Giant-cell arteritis: a review of 35 cases. Quarterly Journal of Medicine, 28, 471-489.

Sunderland, S. (1945). Blood supply of the sciatic nerve and its popliteal divisions in man. Archives of Neurology and Psychiatry (Chic.), 54, 283-289.

Wilson, S. A. K. (1954). Neurology, 2nd edn, vol. 1, p. 111. Edited by A. N. Bruce. Butterworth: London.

Yao, S. T., Goodwin, D. P., and Kenyon, J. R. (1970). Case of ergot poisoning. British Medical Journal, 3, 86-87. 